\title{
Multivariate Analysis of Psychosomatic Complaints as Predictors of Conduct Problems in Swedish Adolescents
}

Samantha Jane Brooks ( $\nabla$ s.j.brooks@ljmu.ac.uk)

Liverpool John Moores University - City Campus: Liverpool John Moores University https://orcid.org/0000-0002-9146-6257

Olga E Titova

Uppsala University: Uppsala Universitet

Emma Ashworth

Liverpool John Moores University - City Campus: Liverpool John Moores University

Simon BA Bylund

Uppsala University: Uppsala Universitet

Inna Feldman

Uppsala University: Uppsala Universitet

Helgi B Schiöth

Uppsala University: Uppsala Universitet

\section{Research article}

Keywords: Conduct Disorder, Psychosomatic Complaints, Adolescence, Multivariate Analysis

Posted Date: June 30th, 2021

DOI: https://doi.org/10.21203/rs.3.rs-649722/v1

License: (c) (1) This work is licensed under a Creative Commons Attribution 4.0 International License.

Read Full License 


\section{Abstract}

Background: Conduct disorders may be linked to common physical conditions in childhood and adolescence, and are often under reported during mainstream school years, which may lead to more serious mental health concerns in adulthood. The aim of the study was to examine the link between specific psychosomatic complaints and adolescent conduct problems.

Methods: 3,132 Swedish adolescents (age range 15-18 years, 47\% boys) completed the Uppsala Life and Health Cross-Sectional Survey (LHS) at school. LHS question scores were grouped in alliance with DSM-5 conduct disorder criteria and psychosomatic complaints (PSC). Multivariate analyses assessed the effects of PSC, age, and gender on conduct problem scores.

Results: Main effects of gender; age; and PSC were observed. Adolescents with higher PSC scores had higher conduct problem scores. In reference to DSM-5 conduct disorder sub-categories, gender, age, and self-reported PSC were associated with increased conduct problems across all domains. Specifically, boys had higher serious violation of rules scores than girls, particularly older boys with higher PSC scores.

Conclusions: These findings suggest that measures of psychosomatic complaints can be used to identify children at risk of developing conduct disorders in the future, to help raise the likelihood of a healthy life into adulthood.

\section{Background}

Mental health difficulties in children and adolescents are increasing, with estimated prevalence rates between $10-20 \%$ worldwide (1). Conduct disorders (CD) are a type of mental health difficulty in young people (2), with $3-4 \%$ of adolescent boys and $1-2 \%$ of adolescent girls receiving a diagnosis (3). While many young children will present mild behavioral difficulties during development, $C D$ is diagnosed when a child's behavior becomes difficult and challenging outside the norm for the age and level of development (4). According to the fifth Edition of the Diagnostic and Statistical Manual for mental disorders (DSM-5) (5), a diagnosis of CD occurs if a child or adolescent meets three out of 15 criteria for disordered behavior over a 12 month period in four categories: aggression to people/animals, deceit and theft, destruction of property and serious violation of rules (e.g. school truancy or prolonged absences from home). Furthermore, age of onset and limited prosocial emotions (LPE, or callous/unemotional traits) are CD specifications recently added to DSM-5 that further determine severity $(6,7)$. As such, using the DSM- 5 criteria as a guide for determining behavioural problems in otherwise typical school children may identify early risk for future, more serious mental health difficulties.

$\mathrm{CD}$ is a leading cause of referral to child and adolescent mental health services, and approximately $60 \%$ of adults with serious mental health problems were diagnosed with adolescent CD (8). Indeed, CD has a significant negative impact on functioning and quality of life, with half of children diagnosed thought to be missing out on key parts of their childhood (2). However, in spite of this, CD is the least understood 
and studied psychiatric disorder and is often over-looked in children and adolescents (8). Therefore, more research is needed to determine how CD criteria are associated with earlier behavioural problems in otherwise healthy school-age children. In turn, this will help to identify those at-risk of later disorders, thus enabling early intervention strategies that can prevent these problems from escalating to diagnosable levels of difficulties.

Examining factors that can predict later conduct problems may also help with early identification. For instance, while it has previously been established that certain psychological and personality factors, such as aggression and callous/unemotional traits can predict the onset of CD (7), there is less evidence for the role of psychosomatic complaints (PSC) in the onset of conduct problems. In some research, PSCs, including headache/migraine, stomach ache, backache, fatigue, anxiety and depression (9) are suggested to precede behavioral problems, such as disengagement with school and home rules, and oppositional/defiant behaviours (10). Similarly, in a recent Swedish multivariate study across 2000 schools, 60,000 young adolescents' experiences of bullying and their mental health complaints were examined (11). The study found that being the perpetrator of bullying predicted a higher incidence of PSCs and behavioural problems, suggesting a relationship between the two. In addition, a large longitudinal study of adolescents across a 23-year period found that self-reported PSCs translated into deterioration - over the study period - of cognitive-behavioural health (12). In terms of specific PSCs, a study of 5730 adolescents reported that headaches were the most common physical complaint and were significantly associated with being the instigator of bullying (13).

A study of younger children found that victims of bullying were at higher risk not only of later $C D$, but also PSCs such as sleep problems, and feeling tense, tired or dizzy (14). However, much of this research has explored the role of bullying on PSCs and conduct problems, and while bullying may be associated with certain domains of $C D$, studies into bullying do not directly examine the link between PSC and conduct problems. Further research may highlight a specific link between PSCs and the potential for future CD diagnosis. Furthermore, it is not yet clear whether behavioral problems reported in school-age children without a formal CD diagnosis are also significantly related to PSCs.

To the authors' knowledge, no large multivariate analysis has yet examined whether specific self-reported adolescent behavioural problems can be related to formal DSM- 5 CD criteria, and in turn whether these specific behavioural problems are associated with a higher incidence of self-reported PSC. In light of this, the aim of the present study was to explore this in a Swedish sample, utilising the Uppsala Life and Health Young Survey (LHS) commissioned by Uppsala County Council, Sweden, between 2005 and 2011 and conducted in school-age children aged 15-19 years. Survey questions were examined by two independent researchers in order to link the LHS questions to behaviours that closely relate to DSM- 5 CD criteria and PSC. It was hypothesized that: a) high levels of self-reported PSC would be related to increased self-reported conduct problems; b) gender differences would be observed in the incidence of PSC and conduct problems, and c) younger versus older adolescents would have significantly different rates of PSC and conduct problems. 


\section{Method}

\section{Design and Participants}

An initial cross-sectional sample of 39,399 adolescents aged 12-19 years was invited to participate anonymously and voluntarily in the Life and Health Young Cross-sectional Survey (LHS), commissioned by the Uppsala County Council, Sweden to be conducted in schools in separate cohorts in 2005, 2007, 2009 , and 2011. All respondents were asked to complete the survey once in one of these years, during school hours. In 2005 and 2007, data on age, household structure and drug use were not available, and so only years 2009 and 2011 were included in the current study. As such, a total of 9,667 adolescents were initially eligible for the present analysis. From this total, a further 99 adolescents were excluded for not having all data for age, gender and PSC, leaving a total of 9,568. Secondary exclusions of 6,436 participants were due to at least one missing answer on questions contributing to the total conduct problems score that was determined by related DSM- 5 CD criteria (described below), leaving a total of 3,132 for the main analyses. The flowchart of the study population exclusions is shown in Fig. 1. The data analysis was approved by the Ethical Committee of Uppsala (EPN).

\section{Measures}

Demographic data. Age, gender, living location (urban or rural), ethnicity (Swedish, Nordic, Other European, Outside Europe), people with whom the adolescents were living, type of living place, and parents' employment, were recorded in the LHS.

Self-reported conduct problems. Conduct problem scores were created by summing scores for questions with Likert-scale or yes/no questions that two independent researchers linked to DSM-5 CD criteria, as summarised in Table 1. Thus, higher conduct problem scores were indicative of higher levels of behaviours typically associated with a diagnosis of CD. Inclusion of questions from the LHS for each CD criteria was checked and agreed independently by two researchers (SB and SBA). Details of each of the included LHS questions (with reference numbers) and their scoring (some scores were reversed by the authors to ensure that high scores reflect high level of conduct problems, as shown by the reference ' $R$ ') are given in Table 2.

DSM-5 CD criterion: Aggression to people or animals. 11 questions were selected from the total LHS to closely represent this criterion, with a cumulative minimum score of 11 and a maximum score of 49 (based on their Likert Score values, see Table 2), including questions such as: Have you exposed someone else to bullying, threats or harassment via mobile or SMS? Have you beaten, kicked or exposed someone else to violence during the school term? Have you participated in fights at school? Have you illegally carried a knife or other weapon in school? Answers included: No, One Time, Many Times, and Never, or One Time, 2-4 times, 5-10 times, more than 10 times.

DSM-5 CD criterion: Destruction of property. Only 2 questions from the LHS were closely matched to this criterion, with a cumulative minimum score of 2 and a maximum of 10 and included the questions: Have you deliberately destroyed or been involved in destroying things? And, have you been involved in 
scribbling, graffiti painting or writing something with a marker or spray paint without permission? Answers included: Never, one time, 2-4 times, 5-10 times or more than 10 times.

DSM-5 CD criterion: Deceitfulness or theft. 9 questions from the LHS closely matched this criterion, with a cumulative minimum score of 9 and maximum of 45, and included the questions: Have you taken money from home or a schoolmate? Have you been involved in a burglary? Have you stolen a bike, or a car? Answers included: Never, one time, 2-4 times, 5-10 times or more than 10 times.

DSM-5 CD criterion: Serious violations of rules. 10 questions from the LHS closely matched this criterion, with a cumulative minimum score of 10 and a maximum of 41, and included questions such as: How easy is it for you at home to say where you go? And, are you truant from school? And, have you not attended some of your subjects? Answers included: Many times, sometimes, not so many times, never. Or, No, not in any subject, Yes 1-2 subjects, Yes, 3-4 subjects, Yes, more than 5 subjects.

Total conduct problems score. All 32 questions from the categories above were summed to create a cumulative LHS minimum score of 32 and a maximum score of 145 (according to the Likert Scale scores for each question). Percentages for total LHS scores and the 4 categories (e.g. according to the highest possible score) were calculated for contrast homogeneity.

Assessment of self-reported psychosomatic complaints (PSC). 12 LHS questions related to psychological and 12 related to somatic (physical) complaints, which were again checked by the same two independent researchers (SB, SBA). Percentages for PSC total and subscale scores (e.g. according to the highest possible score for total Likert Scale scores) were calculated for contrast homogeneity. Three levels of PSC: low, medium and high were calculated as tertile thresholds of the total PSC percentage scores. See Table 3 for details of each of the included questions and their scoring (some scores are reversed to ensure that high scores denote high level of PSC).

Psychological complaints. The 12 psychological LHS questions have a cumulative minimum score of 12 and a maximum score of 53 and are related to: stress, nervousness, anxiety/worry, depression, happiness, medication for anxiety/depression/sleep disorder, reading/writing difficulties, neuropsychiatric disorder (e.g. ADHD), worries about sleep, occurrence of nightmares, and how bright the future looks. Answers include: Never, rarely, sometimes, often, always.

Somatic complaints. The 12 somatic (physical) LHS questions have a cumulative minimum score of 12 and a maximum score of 123 and are related to: how the person feels, incidence of migraine, headache, stomach ache, ringing in the ears/tinnitus, tiredness, pain in the neck and shoulders, pain in the back and hips, pain in the hands, knees, legs or feet, quality of dental health, whether prescription or nonprescription drugs are taking for headaches or other pain, hearing loss, visual impairment that cannot be correct with glasses, physical disability, difficulty sleeping, and whether the person is often tired. Answers include: never, rarely, sometimes, often and always. Total PSC score. All 24 LHS questions above were summed to create a cumulative minimum score of 24 and a maximum score of 176 , which allowed for the calculation of individual total percentage PSC scores. 


\section{Statistical analyses}

SPSS version 25.0 (IBM Corp, 2017) was used. Descriptive statistics of the demographic data were conducted with t-tests for mean differences and chi-squared for frequencies, where applicable. Bonferroni correction was applied to control for multiple comparisons, and parametric assumptions (normal distribution, homogeneity of variance, sphericity and collinearity) were checked and data corrected if these assumptions were violated. A p-value of equal to or less than 0.05 denoted significant effects.

Analysis of variance (ANOVA) of total conduct problems score $(n=3,132)$. A 3-way independent measures design, incorporating the between-subject factors: age (dichotomised above and below the mean age of 16 years), gender (boys, girls) and PSC severity score (tertiles for low, medium, high) was utilised to examine main effects and interactions associated with variance in the total LHS conduct problem score.

Multivariate analysis of variance (MANOVA) of the 4 categories of conduct problems $(n=3,132)$. A 3-way independent measures design, incorporating the between-subject factors as above, was utilised to examine the main effects and interactions associated with score variance in the four conduct problem categories that were linked to DSM-5 CD namely: a) aggression to people/animals, b) deceit/theft, c) destruction of property, and d) serious violations of rules.

\section{Results}

\section{Demographics}

A total of 1460 boys and 1672 girls contributed to the ANOVA and MANOVA analyses in a total sample of 3,132 adolescents after exclusions. According to a mean age split at 16 years of age, 1533 were 'younger adolescents' (15-16 years of age) and 1599 were 'older adolescents' (17-18 years of age). More adolescents lived in urban (Uppsala City) as opposed to rural communities $(p<0.001)$, and there were more adolescents of Swedish nationality than other Nordic, European or foreign nationalities in the sample $(p<0.001)$. Most lived with both parents in a townhouse owned by their family, with most having parents in employment (see Table 4).

1321 adolescents were rated as having low-, 868 with medium- and 943 with high-PSC severity. No significant difference in age was detected in the sample of boys and girls. Adolescent girls reported more high PSCs than boys, specifically, they reported a higher severity of headaches, stomach aches and tiredness, as well as higher levels of anxiety and depression (see Table 4).

\section{Analysis of variance (ANOVA) of total conduct problems score $(n=3,132)$}


The effects of gender (boys, girls), age (younger, older adolescent) and PSC severity (low, medium, high) on total conduct problems score was examined. Significant main effects of gender $(F[1,3000]=124.220$, $\left.p<0.001, \eta^{2}=0.038\right)$; age $\left(F[1,3000]=21.664, p<0.001, \eta^{2}=0.007\right)$; and PSC severity $(F[2,3000]=77.342$, $\left.p<0.001, \eta^{2}=0.046\right)$ were observed. No significant interactions were observed. Post-hoc $t$-tests confirmed that boys had significantly higher total conduct problems scores than girls $(t[2898.579]=8.114, p<$ $0.001)$; older adolescents had higher conduct problems scores than younger $(t[3459]=4.699, p<0.001)$; and all adolescents regardless of age or gender with high PSC severity had higher conduct problems scores, relative to those with medium $(t[2053.938]=7.000, p<0.001)$ or $\operatorname{low}(t[2203.242]=9.333, p<0.001)$ PSC severity. Those with medium PSC severity had higher conduct problems scores than those with low severity $(t[2038]=2.630, p<0.009)$. See Fig. 2 for illustration of scores across gender and age for conduct problems score differences, and Table 5 for total and categories of conduct problems associated with DSM-5 CD criteria.

\section{Multivariate analysis of variance (MANOVA) of the four categories of conduct problems $(n=3,132)$}

The effects of gender (boys, girls), age (younger, older adolescents) and PSC severity (low, medium, high) on categories of conduct problems (aggression to people/animals, deceit/theft, destruction to property, serious violation of rules) were examined. A main effect of gender across all categories was observed $\left(F[1,3000]>1, p<0.001, \eta^{2}=0.011-0.021\right)$, and post-hoc $t$-tests confirmed that boys compared to girls had higher scores for aggression to people/animals $(t[2797.928]=9.770, p<0.001)$, deceit/theft $(t[3163.635]=45.631, p<0.001)$, destruction to property $(t[7493.270]=19.653, p<0.001)$ and serious violation of rules $(t[9095.183]=8.606, p<0.001)$. Only a main effect of age on the incidence of serious violations of rules was observed $\left(F[4,3000]=42.751, p<0.001, \eta^{2}=0.051\right)$, and post-hoc $t$-tests confirmed that older compared to younger adolescents committed significantly more serious violations of rules $(t[8770.816]=21.658, p<0.001)$. A main effect of PSC severity across all subtypes of conduct problems was observed $\left(F[2,3000]>1, p<0.001, \eta^{2}=0.005-0.022\right)$. Post-hoc t-tests confirmed that higher conduct problem scores across all subtypes were observed in adolescents with a high and medium compared to low PSC severity score $(P<0.003)$.A significant interaction between gender $x$ PSC severity was found on the serious violation of rules subtype $\left(F[2,3000]=2.897, p=0.05, \eta^{2}=0.002\right)$, and post-hoc t-tests confirmed that boys had higher serious violation of rules scores than girls $([19095.183]=8.606, p<0.001)$. Also, boys with higher PSC severity scores had higher serious violation of rules scores that those with medium $(t[749.177]=3.842, p<0.001)$ or low $(t[589.497]=7.804, p<0.001)$ PSC scores; and those with medium PSC scores had higher serious violation of rules scores than those with low PSC scores $(t[1068.563]=4.507, p<0.001)$. See Fig. 3 .

\section{Discussion}

To the best of our knowledge, this is the first study to show that in an otherwise healthy population of Swedish adolescents attending mainstream school, high levels of PSCs are associated with higher levels 
of total conduct problems (linked to DSM-5 CD criteria). In particular, this association was amplified for boys who scored highly in the serious rule violations category, with those with higher PSC severity scores displaying higher levels of serious rule violations. In addition, consistent with previous research, older adolescents (17-18 years of age) reported higher levels of PSCs and conduct problems than younger adolescents (15-16 years of age), and boys reported significantly higher levels of conduct problems than girls.

The finding that adolescents in this sample, particularly boys aged 17-18 years, exhibit higher levels of non-aggressive executive dysfunction in the form of serious rule violations, associated with increasing PSC, provides some clues as to the potential underlying factors associated with conduct problems. Nonaggressive - or cool - executive dysfunction refers to cognitions such as planning, flexible thinking and working memory (8), which contribute to an ability to adhere to societal rules (e.g. remembering to turn up to class, planning to return home, empathising with those setting the rules). Moreover, cool executive dysfunction is also associated with adolescent attention deficit hyperactivity disorder (15)'(16), suggesting a common aetiology underpinning the different types of behavioural disorders. Furthermore, cool executive dysfunction has been related to lower brain activation in the precuneus, superior temporal, dorsal anterior cingulate cortex, and insula in response to emotional stimuli compared to healthy controls, areas of the brain broadly related to goal-planning and empathising (17). These findings suggest that increased somatic complaints early in life may contribute to altered development in neural circuitry associated with top-down emotion processing and self-regulation, leading to a propensity towards conduct problems.

Conversely, altered neural development may contribute to low prosocial emotion/callous unemotional traits that increase the risk of having conduct problems $(6,7)$. In addition, effective emotion regulation and adaptive decision-making relies on somatic processing (e.g. the somatic marker hypothesis), or a 'gut feeling', which is necessary for cooler executive functions, including empathy, understanding future consequences of actions, and the learning of beneficial decisions that adhere to societal rules (18). As such, gauging and addressing common adolescent PSCs, including frequent headaches, stomach aches, tiredness, anxiety and depression, may reduce the risk of conduct problems - particularly in boys - and the future development of adult mental health disorders, such as antisocial personality disorder (common in males), anxiety and depression (common in females) and other psychopathologies (19). Together with previous literature, our findings suggest that conduct problems may have at least part of their origin in being mentally and/or physically unwell with PSCs during childhood and adolescence.

Specific PSCs, namely stomachache, tiredness and problems related to anxiety were associated with selfreported conduct problems in the present study. However, while this association was strongest for boys, it was girls reporting higher PSC scores. This may be explained by the measure of mental health difficulties used (i.e., conduct problems). It is possible that there is a gender difference in the presentation of mental health difficulties or in coping strategies for PSCs between boys and girls. For instance, while PSCs were associated with conduct problems in the current study, previous research has also found that childhood somatic complaints can predict generalized anxiety and depression in adolescents (20). As boys are 
more likely to experience externalising difficulties (such as behaviour problems explored here), and girls are more likely to present with internalising difficulties (such as anxiety and depression) $(21,22)$, this may explain the stronger association between PSCs and conduct problems in boys in the present study. It may be that PSCs contribute equally to mental health difficulties, but the way these difficulties manifest themselves differs by gender. If this is the case, had an internalising measure of mental health difficulties been used, the gender effect may have been reversed. Future research could seek to further explore this, investigating gender differences in the relationship between PSCs and types of mental health difficulties.

Finally, a striking point about the findings of this study is that the sample were not adolescents with a formal DSM-5 CD diagnosis, but were otherwise healthy and attending mainstream school. Thus, the relationships observed in the present study may be even stronger in individuals with a diagnosis of CD. Furthermore, the results from the present study highlight the relatively higher levels of conduct problems and PSC reported by adolescents in a general population sample, which may remain untreated if undiagnosed. This is problematic for individuals and society, given that adolescents with recurring behavioural problems are $60 \%$ more likely to develop serious mental health disorders, such as anti-social personality disorder, in adulthood (8). As such, these data provide further motivation to reverse the present trend that CD remains the least understood and studied psychiatric disorder(8).

\section{Strengths and limitations}

One of the main strengths of this study is the large sample size, and the calculation of scores from a large range of questions that were confidentially and anonymously answered by adolescents at school. In addition, two researchers (SB and SBA) independently linked the LHS questions to DSM-5 CD criteria. However, limitations include the reliance on adolescent self-report measures of behaviour that were not formally related to DSM- 5 CD criteria, which may not be as accurate as behavioural observations or the use of validated CD measures. Furthermore, this was a cross-sectional as opposed to a longitudinal study, and so conclusions regarding the differences in age, and causal relationships, must be considered cautiously. Moreover, it is imperative to collect more recent data, as the last data in this sample was taken in 2011. Finally, while efforts were taken by the authors to closely match the LHS questions to DSM-5 CD criteria, the survey was not a validated measure of CD.

\section{Conclusions}

The present study aimed to better understand adolescent conduct problems. We considered the relationship between self-reported PSC and conduct problems, to determine whether PSCs are a valuable gauge for early interventions to prevent the development of serious mental disorders into adulthood. The findings we present suggest that high levels of PSCs are associated with higher levels of total conduct problems, and each of the four conduct problems subtypes (linked to DSM-5 CD criteria). In particular, this association is amplified for boys who score highly in the serious rule violations category, with those with higher PSC severity scores displaying higher levels of serious rule violations. The hot versus cold executive dysfunction model of conduct disorder may provide clues as to how early life PSCs may 
influence brain development in neural processes associated with decision-making, emotion regulation and empathy. An inability to effectively utilise somatic cues (e.g. due to a higher level of pervasive PSC) may increase the prevalence of low prosocial emotion, rule violation, anxiety and depression.

Furthermore, when combining the findings from the present study with previous research, it is possible that PSCs contribute to mental health difficulties in both genders, but that the coping mechanisms or presentation of symptoms differ between boys and girls. This is a crucial direction for future research. These data suggest that child and adolescent screening at school for psychosomatic complaints may help to identify the need for intervention that prevents future development of behavioural problems and adult psychiatric conditions.

\section{List Of Abbreviations}

ADHD Attention Deficit Hyperactivity Disorder

ANOVA Analysis of Variance

CD Conduct Disorder

DSM-5 Diagnostic and Statistical Manual of Mental Disorders V.5

LHS Uppsala Life and Health Cross-sectional survey

MANOVA Multivariate Analysis of Variance

PSC Psychosomatic Complaints

SPSS Statistical Package for the Social Sciences

\section{Declarations}

Ethics approval and consent: The data analysis was approved by the Ethical Committee of Uppsala (EPN).

\section{Consent for publication: N/A}

Availability of data and materials: The datasets used and/or analysed during the current study are available from the corresponding author on reasonable request. SJB had full access to all data and takes responsibility for the integrity and accuracy of the data analysis.

Competing interests: The authors declare that they have no competing interests

Funding: HBS is supported by the Swedish Research Foundation and the Swedish Brain Research foundation. The funding sources had no role in the design and conduct of the study; collection, 
management, analysis, and interpretation of the data; and preparation, review, or approval of the manuscript.

Authors' contributions: SJB analysed the data and wrote the first draft of the paper; OET prepared the data and managed the dataset, and contributed to substantial revisions of the manuscript; EA contributed to revisions of the manuscript and aided interpretation of the data, SBAB cross-referenced the coding of the main variables into DSM5 CD-comparable categories; IF contributed to substantial revisions of the manuscript and aided interpretation; HBS shared the data and research assistance, and contributed to revisions of the manuscript.

Acknowledgements: We thank Uppsala County Council for providing anonymous data from the "Life and Health Young Cross-Sectional Survey", and we thank Dr PH for her initial work with this sample.

\section{References}

1. Organisation WH. WHO. https://www.who.int/. 2020.

2. 158 NCGN. Antisocial behaviour and conduct disorders in children and young people: recognition and management. (UK) NIfHaCE, editor. London UK: NICE; 2017.

3. Polanczyk GV, Salum GA, Sugaya LS, Caye A, Rohde LA. Annual research review: A meta-analysis of the worldwide prevalence of mental disorders in children and adolescents. J Child Psychol Psychiatry. 2015;56(3):345-65. doi:10.1111/jcpp.12381

4. Ogundele MO. Behavioural and emotional disorders in childhood: A brief overview for paediatricians. World J Clin Pediatr. 2018;7(1):9-26. doi:10.5409/wjcp.v7.i1.9

5. Association AP. Diagnostic and Statistical Manual of Mental Disorders. Fifth ed: American Psychiatric Press Inc.; 2013.

6. Jambroes T, Jansen LM, Vermeiren RR, Doreleijers TA, Colins OF, Popma A. The clinical usefulness of the new LPE specifier for subtyping adolescents with conduct disorder in the DSM 5. Eur Child Adolesc Psychiatry. 2016;25(8):891-902. doi:10.1007/s00787-015-0812-3

7. Kohls G, Baumann S, Gundlach M, et al. Investigating Sex Differences in Emotion Recognition, Learning, and Regulation Among Youths With Conduct Disorder. J Am Acad Child Adolesc Psychiatry. 2019. doi:10.1016/j.jaac.2019.04.003

8. Fairchild G, Hawes DJ, Frick PJ, et al. Conduct disorder. Nat Rev Dis Primers. 2019;5(1):43. doi:10.1038/s41572-019-0095-y

9. Murberg TA BE. School-related stress and psychosomatic symptoms among Norwegian adolescents. School Psychology International 2004;25(3):317-32.

10. Viner RM, Ozer EM, Denny S, et al. Adolescence and the social determinants of health. Lancet. 2012;379(9826):1641-52. doi:10.1016/S0140-6736(12)60149-4

11. Modin B, Plenty S, Laftman SB, et al. School Contextual Features of Social Disorder and Mental Health Complaints-A Multilevel Analysis of Swedish Sixth-Grade Students. Int J Environ Res Public 
Health. 2018;15(1). doi:10.3390/ijerph15010156

12. van Geelen SM, Hagquist C. Are the time trends in adolescent psychosomatic problems related to functional impairment in daily life? A 23-year study among 20,000 15-16year olds in Sweden. J Psychosom Res. 2016;87:50-6. doi:10.1016/j.jpsychores.2016.06.003

13. Vaiciunas T, Smigelskas K. The Role of School-Related Well-Being for Adolescent Subjective Health Complaints. Int J Environ Res Public Health. 2019;16(9). doi:10.3390/ijerph16091577

14. Gini G. Associations between bullying behaviour, psychosomatic complaints, emotional and behavioural problems. Journal of Paediatrics and Child Health. 2008;44(9):492-7.

15. Hobson CW, Scott S, Rubia K. Investigation of cool and hot executive function in ODD/CD independently of ADHD. J Child Psychol Psychiatry. 2011;52(10):1035-43. doi:10.1111/j.14697610.2011.02454.x

16. Dolan M, Lennox C. Cool and hot executive function in conduct-disordered adolescents with and without co-morbid attention deficit hyperactivity disorder: relationships with externalizing behaviours. Psychol Med. 2013;43(11):2427-36. doi:10.1017/S0033291712003078

17. Noordermeer SD, Luman M, Oosterlaan J. A Systematic Review and Meta-analysis of Neuroimaging in Oppositional Defiant Disorder (ODD) and Conduct Disorder (CD) Taking Attention-Deficit Hyperactivity Disorder (ADHD) Into Account. Neuropsychol Rev. 2016;26(1):44-72. doi:10.1007/s11065-015-9315-8

18. Blair RJ. Neurocognitive models of aggression, the antisocial personality disorders, and psychopathy. J Neurol Neurosurg Psychiatry. 2001;71(6):727-31. doi:10.1136/jnnp.71.6.727

19. Sobhani M, Bechara A. A somatic marker perspective of immoral and corrupt behavior. Soc Neurosci. 2011;6(5-6):640-52. doi:10.1080/17470919.2011.605592

20. Shanahan L, Zucker N, Copeland WE, Bondy CL, Egger HL, Costello EJ. Childhood somatic complaints predict generalized anxiety and depressive disorders during young adulthood in a community sample. Psychol Med. 2015;45(8):1721-30. doi:10.1017/S0033291714002840

21. Leadbeater BJ KG, Hertzog C, Blatt SJ. A multivariate model of gender differences in adolescents' internalising and externalising problems. Developmental Psychology. 1999;35(5):1268-82.

22. Carragher N, Krueger RF, Eaton NR, Slade T. Disorders without borders: current and future directions in the meta-structure of mental disorders. Soc Psychiatry Psychiatr Epidemiol. 2015;50(3):339-50. doi:10.1007/s00127-014-1004-z

\section{Tables}

Due to technical limitations, table 1 to 5 PDF's are only available as a download in the Supplemental Files section.

\section{Figures}




\section{Flow Diagram of Exclusions}

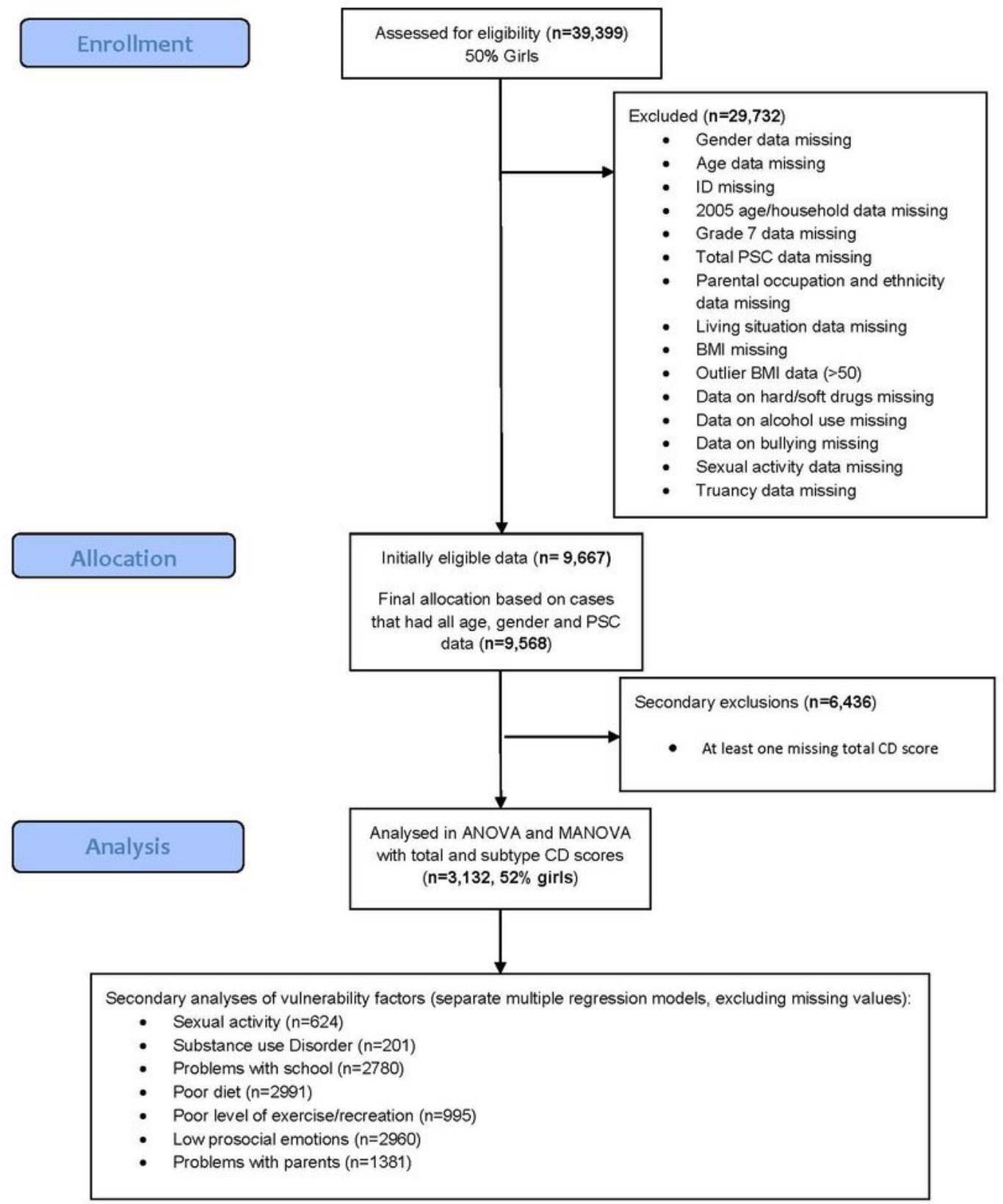

\section{Figure 1}

Flow Diagram of Exclusions 


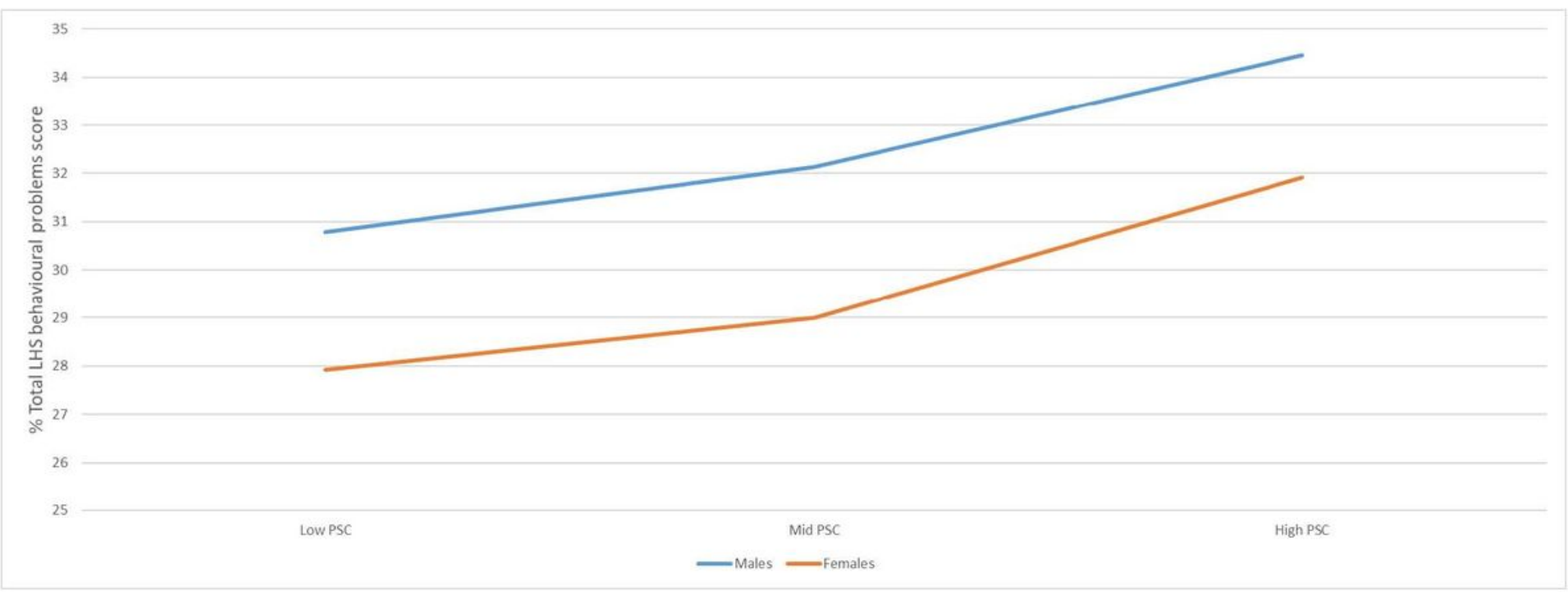

\section{Figure 2}

Total LHS behavioural scores and the relationship to increasing levels of self-reported psychosomatic complaints (percentage of the total sum of Likert Scale questionnaire responses) in Swedish adolescents aged 15-19 years of age. PSC=Psychosomatic complaint score based on questions from the Uppsala Life and Health Survey (LHS); \% score is the total behavioural disorder score calculated from maximum Likert Scale scores.

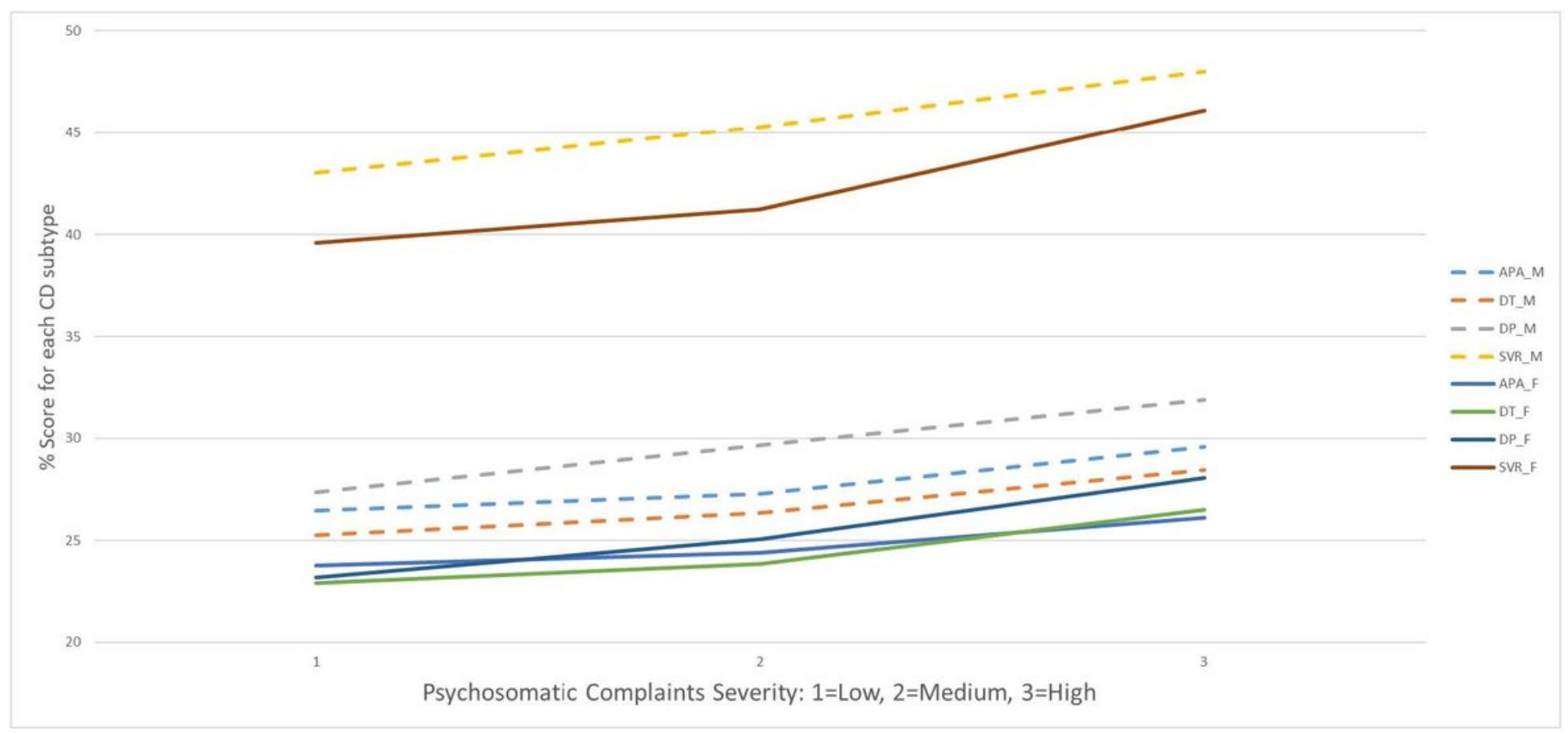

\section{Figure 3}

LHS behavioural problem scores mapped onto DSM5 Conduct Disorder criteria (confirmed by two independent researchers) in relation to levels of psychosomatic complaints in Swedish adolescents aged 15-19 years old. Legend: APA=Aggression to people/animals; DT=Deceit/theft; DP=Destruction of 


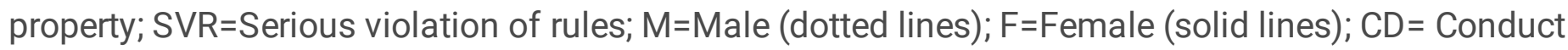
Disorder; DSM5=Diagnostic and Statistical Manual of Mental Disorders version 5.

\section{Supplementary Files}

This is a list of supplementary files associated with this preprint. Click to download.

- Table1DSMConductDisorderCriteria.pdf

- Table2LifeandhealthsurveyquestionsandDSM5CD.pdf

- Table3PsychosomaticComplaintssurveyquestions.pdf

- Table4Demographics.pdf

- Table5MeanCDScores.pdf 